\title{
TVERBERG-TYPE THEOREMS FOR INTERSECTING BY RAYS
}

\author{
R.N. KARASEV
}

\begin{abstract}
In this paper we consider some results on intersection between rays and a given family of convex, compact sets. These results are similar to the center point theorem, and Tverberg's theorem on partitions of a point set.
\end{abstract}

\section{INTRODUCTION}

In this paper we consider some results on intersection between rays and a given family of convex, compact sets, that resemble the center point theorem of [18, 19], and Tverberg's theorem on partitions from [23].

Let us make a definition. Consider a straight line $\ell \subset \mathbb{R}^{d}$ and a point $p \in \ell$. The point $p$ divides $\ell$ into two half-lines, we call these half-lines rays starting at $p$. We are going to study the questions of the following type: given a family $\mathcal{F}$ of convex sets in $\mathbb{R}^{d}$, find a point $p \in \mathbb{R}^{d}$ such that every ray starting at $p$ intersects at least $\alpha|\mathcal{F}|$ members of $\mathcal{F}$, or at most $\beta|\mathcal{F}|$ members of $\mathcal{F}$. Such questions were considered before in [20, 10], for the case of hyperplanes, and in [5, 11] for families of convex sets.

The following theorem is similar to the "dual" Tverberg theorem for hyperplanes from [10], the statements of this kind (with minor differences) for hyperplanes were conjectured in [20].

Theorem 1. Let $\mathcal{F}$ be a family of $n$ compact convex sets in $\mathbb{R}^{d}$, such that any point $x \in \mathbb{R}^{d}$ belongs to at most $c$ sets of $\mathcal{F}$. Suppose that $r$ is a prime power and the following inequality holds

$$
n \geq(d+1)(r-1)+c+1 .
$$

Then $\mathcal{F}$ has $r$ disjoint subfamilies $\mathcal{F}_{1}, \ldots, \mathcal{F}_{r}$, such that there exists a point $p \in \mathbb{R}^{d}$ with the following property: for any ray $\rho$ starting at $p$, and any subfamily $\mathcal{F}_{i}$, there exists $K \in \mathcal{F}_{i}$ such that $\rho \cap K=\emptyset$.

The following theorem is a generalization of the result of [5], see also [20], where a particular case was conjectured for families of hyperplanes. This is an analogue of the central point theorem for finite point sets, see [18, 19, 6].

Corollary 2. Let $\mathcal{F}$ be a family of $n$ compact convex sets in $\mathbb{R}^{d}$, such that any point $x \in \mathbb{R}^{d}$ belongs to at most $c$ sets of $\mathcal{F}$. Suppose that $r$ is a positive integer and the

2000 Mathematics Subject Classification. 52A20, 52A35, 52C35.

Key words and phrases. central point theorem, Tverberg's theorem, Helly's theorem.

This research is supported by the Dynasty Foundation, the President's of Russian Federation grant MK-113.2010.1, the Russian Foundation for Basic Research grants 10-01-00096 and 10-01-00139. 
following inequality holds

$$
n \geq(d+1)(r-1)+c+1 .
$$

Then there exists a point $p \in \mathbb{R}^{d}$ such that any ray $\rho$ starting at $p$ does not intersect at least $r$ of the sets in $\mathcal{F}$.

Theorem 1 is formulated for compact sets, and the compactness is essential in the proof. Still, it is possible to formulate a similar result for hyperplanes. Let us make some definitions.

Definition 1. A convex open subset $G \subset \mathbb{R}^{d}$ is called almost bounded, if it does not contain an open cone. Equivalently, for any point $p \in G$ the set of rays starting at $p$, and lying within $G$, has an empty interior as a subset of the unit sphere $S^{n-1}$.

Definition 2. For a family of hyperplanes $\mathcal{G}$ in $\mathbb{R}^{d}$ denote by $C(\mathcal{G})$ the union of all almost bounded components of the complement $\mathbb{R}^{d} \backslash \cup \mathcal{G}$.

The following theorem generalizes the dual Tverberg theorem from [10] to the case, when hyperplanes are not in general position. This statement is also a partial solution of Conjecture 2 in [20].

Theorem 3. Let $\mathcal{F}$ be a family of $n$ hyperplanes in $\mathbb{R}^{d}$, such that any point $x \in \mathbb{R}^{d}$ belongs to at most c hyperplanes of $\mathcal{F}$. Suppose that $r$ is a prime power and the following inequality holds

$$
n \geq(d+1)(r-1)+c+1 .
$$

Then $\mathcal{F}$ has $r$ disjoint subfamilies $\mathcal{F}_{1}, \ldots, \mathcal{F}_{r}$, such that

$$
\bigcap_{i=1}^{r} C\left(\mathcal{F}_{i}\right) \neq \emptyset .
$$

The proofs in this paper mostly follow the proofs in [10], the essential difference is that the general position requirements are substituted by an upper bound of the covering multiplicity of a family. Such strengthening is allowed by an accurate use of the concept of the Krasnosel'skii-Schwarz genus (see Section 4 for the definition) to avoid singular configurations that give a solution of the topological problem (in terms of sections of a vector bundle), but do not correspond to the solution of the original geometric problem.

\section{FACTS FROM TOPOLOGY}

In this section some topological facts, that arise in the proof of Theorem 1 are given. In fact, the first part of the proof follows the proof of Theorem 1.1 in [11], this and the following sections restate the needed lemmas.

We consider topological spaces with continuous (left) action of a finite group $G$ and continuous maps between such spaces that commute with the action of $G$. We call them $G$-spaces and $G$-maps. In this paper we actually consider groups $G=\left(Z_{p}\right)^{k}$ for prime $p$, called usually $p$-tori, but most of the definitions are valid for arbitrary finite group $G$.

For basic facts about (equivariant) topology and vector bundles the reader is referred to the books [9, 14, 17]. The cohomology is taken with coefficients $Z_{p}$ ( $p$ is the same as in 
the definition of $G$ ), in notations we omit the coefficients. Let us start from some standard definitions.

Definition 3. Denote by $E G$ the classifying $G$-space, which can be thought of as an infinite join $E G=G * \cdots * G * \ldots$ with diagonal left $G$-action. Denote $B G=E G / G$. For any $G$-space $X$ denote $X_{G}=(X \times E G) / G$, and put (equivariant cohomology in the sense of Borel) $H_{G}^{*}(X)=H^{*}\left(X_{G}\right)$. It is easy to verify that for a free $G$-space $X$, the space $X_{G}$ is homotopy equivalent to $X / G$.

Consider the algebra of $G$-equivariant cohomology of the point $A_{G}=H_{G}^{*}(\mathrm{pt})=H^{*}(B G)$. For a group $G=\left(Z_{p}\right)^{k}$, the algebra $A_{G}=H_{G}^{*}\left(Z_{p}\right)$ has the following structure (see [9]). In the case $p$ odd it has $2 k$ multiplicative generators $v_{i}, u_{i}$ with dimensions $\operatorname{dim} v_{i}=1$ and $\operatorname{dim} u_{i}=2$ and relations

$$
v_{i}^{2}=0, \quad \beta v_{i}=u_{i} .
$$

We denote by $\beta(x)$ the Bockstein homomorphism.

In the case $p=2$ the algebra $A_{G}$ is the algebra of polynomials of $k$ one-dimensional generators $v_{i}$.

Any representation of $G$ can be considered as a vector bundle over the point pt, and it has corresponding characteristic classes in $H_{G}^{*}(\mathrm{pt})$. We need the following lemma, that follows from the results of [9], Chapter III $\S 1$.

Lemma 1. Let $G=\left(Z_{p}\right)^{k}$, and let $I[G]$ be the subspace of the group algebra $\mathbb{R}[G]$, consisting of elements

$$
\sum_{g \in G} a_{g} g, \quad \sum_{g \in G} a_{g}=0 .
$$

Then the Euler class $e(I[G]) \neq 0 \in A_{G}$ and is not a divisor of zero in $A_{G}$.

Note that in this lemma the fact that $G=\left(Z_{p}\right)^{k}$ is essential.

\section{Topology of TVerberg's TheOrem}

This paper reproduces some lemmas from [11. In Tverberg's theorem and its topological generalizations (see [2, 24] for example) it is important to consider the configuration space of $r$-tuples of points $x_{1}, \ldots, x_{r} \in \Delta^{N}$ with pairwise disjoint supports. Here $\Delta^{N}$ is a simplex of dimension $N$. Let us make some definitions, following the book [15].

Definition 4. Let $K$ be a simplicial complex. Denote by $K_{\Delta}^{r}$ the subset of the $r$-fold product $K^{r}$, consisting of the $r$-tuples $\left(x_{1}, \ldots, x_{r}\right)$ such that every pair $x_{i}, x_{j}(i \neq j)$ has disjoint supports in $K$. We call $K_{\Delta}^{r}$ the $r$-fold deleted product of $K$.

Definition 5. Let $K$ be a simplicial complex. Denote by $K_{\Delta}^{* r}$ the subset of the $r$-fold join $K^{* r}$, consisting of convex combinations $w_{1} x_{1} \oplus \cdots \oplus w_{r} x_{r}$ such that every pair $x_{i}, x_{j}(i \neq j)$ with weights $w_{i}, w_{j}>0$ has disjoint supports in $K$. We call $K_{\Delta}^{* r}$ the $r$-fold deleted join of $K$. 
Note that the deleted join is a simplicial complex again, while the deleted product has no natural simplicial complex structure, although it has some cellular complex structure.

The $r$-fold deleted product of the simplex $\Delta^{(r-1)(d+1)}$ is the natural configuration space in Tverberg's theorem, but sometimes it is simpler to use the deleted join. Denote by $[r]$ the set $\{1, \ldots, r\}$, with the discrete topology.

If $r$ is a prime power $r=p^{k}$, then the group $G=\left(Z_{p}\right)^{k}$ can be somehow identified with $[r]$, so a $G$-action on $K_{\Delta}^{r}$ and $K_{\Delta}^{* r}$ by permuting $[r]$ arises. The following lemma is well-known, see [24] for example.

Lemma 2. The deleted join of the simplex $\left(\Delta^{N}\right)_{\Delta}^{* r}=[r]^{* N+1}$ is $N$-1-connected, and the natural map $A_{G}^{l} \rightarrow H_{G}^{l}\left(\left(\Delta^{N}\right)_{\Delta}^{* r}\right)$ is injective for $l \leq N$.

Let us say a few words about the proof. There is the Leray-Serre spectral sequence that relates the ordinary cohomology of a $G$-space $X$ to its equivariant cohomology, the bottom row of $E_{2}$ in this spectral sequence being $A_{G}^{*}$. The connectedness hypothesis implies that the corresponding part of the bottom row survives in $E_{\infty}$, that is the statement of the lemma.

The next lemma is used in [24] too, a proof of this lemma can be found in [11], for example.

Lemma 3. Let $r=p^{k}, G=\left(Z_{p}\right)^{k}$, and let $K$ be a simplicial complex. If the natural map $A_{G}^{l} \rightarrow H_{G}^{l}\left(K_{\Delta}^{* r}\right)$ is injective for $l \leq N$, then the similar map $A_{G}^{l} \rightarrow H_{G}^{l}\left(K_{\Delta}^{r}\right)$ is injective for $l \leq N-r+1$.

\section{The Genus of $G$-SPACES}

In this section we describe some measure of complexity for a $G$-space. Let $X$ be a paracompact free $G$-space, $G$ being a finite group. Informally, the main idea is that this measure can be estimated from the equivariant cohomology of $X$, by the statements like those in Lemmas 2 and 3 , Let us make a definition.

Definition 6. The free genus of a free $G$-space $X$ is the least number $n$ such that $X$ can be covered by $n$ open subsets $X_{1}, \ldots, X_{n}$ so that every $X_{i}$ can be $G$-mapped to $G$. Denote the free genus by $g_{\text {free }}(X)$.

There are several kinds of genus for a $G$-space, here we only use the free genus, and call it simply "genus". The free genus was introduced in [13, 21, 22, different versions of this definition for non-free action are discussed in 3 .

Let us explain the definition of the genus. The set $X_{i}$ in the definition can be $G$-mapped to $G$ iff the group $G$ acts on connected components of $X_{i}$ freely, we call such spaces inessential in the sequel. In fact, for paracompact $X$ the sets $X_{i}$ in the definition of genus may be taken closed instead of open.

Let us state the properties of the genus, valid for paracompact spaces, following [25].

(1) (Monotonicity) If there is a $G$-map $f: X \rightarrow Y$, then $\mathrm{g}_{\text {free }}(X) \leq \mathrm{g}_{\text {free }}(Y)$;

(2) (Subadditivity) Let $X=A \cup B$, where $A, B$ are closed or open $G$-invariant subspaces. Then $\mathrm{g}_{\text {free }}(X) \leq \mathrm{g}_{\text {free }}(A)+\mathrm{g}_{\text {free }}(B)$; 
(3) (Dimension upper bound) $\mathrm{g}_{\text {free }}(X) \leq \operatorname{dim} X+1$;

(4) (Cohomology lower bound) If the natural map $A_{G}^{n} \rightarrow H_{G}^{n}(X, M)$ is nonzero for some $G$-module $M$, then $\mathrm{g}_{\text {free }}(X) \geq n+1$.

Take the deleted join $\left(\Delta^{N}\right)_{\Delta}^{* r}$ and the deleted product $\left(\Delta^{N}\right)_{\Delta}^{r}$, considered in the previous section for $r$ being a prime power, with an action of the corresponding $p$-torus. Then the cohomology lower bound and the dimension upper bound, with Lemmas 2 and 3 give

$$
\mathrm{g}_{\text {free }}\left(\left(\Delta^{N}\right)_{\Delta}^{* r}\right)=N+1, \quad \mathrm{~g}_{\text {free }}\left(\left(\Delta^{N}\right)_{\Delta}^{r}\right)=N-r+2 .
$$

We need the following lemma, that can be considered a strengthening of the definition of genus. A particular case of this lemma for $G=Z_{2}$ was proved in [12, Theorem 9].

Lemma 4. Let $X$ be a paracompact $G$-space, let $\mathcal{U}=\left\{U_{i}\right\}_{i=1}^{N}$ be some open (or closed) covering of $X$ by inessential invariant subsets. Then there exist a point $x \in X$, that is covered by at least $\mathrm{g}_{\text {free }}(X)$ sets of $\mathcal{U}$.

Proof. Since every $U_{i}$ can be mapped to $G$, then from the partition of unity, corresponding to $\mathcal{U}$, arises a map $f: X \rightarrow G^{* N}$.

Consider the contrary: the covering $\mathcal{U}$ has multiplicity at most $\mathrm{g}_{\text {free }}(X)-1$. Then the image of $f$ is within the $\left(g_{\text {free }}(X)-2\right)$-dimensional skeleton of $G^{* N}$. Now from the dimension upper bound and the monotonicity of the genus it follows that $g_{\text {free }}(X) \leq \mathrm{g}_{\text {free }}(X)-1$, which is a contradiction.

Note that this lemma is true if we consider the fixed-point-free genus $g_{G}(X)$ (see [3, 25]) of a fixed point free $G$-space, and call a subset inessential if none of its connected components is stabilized by the whole group $G$. This follows from the dimension upper bound for fixedpoint-free genus.

\section{Proof of Theorem 1}

Consider the simplex $\Delta=\Delta^{n-1}$, along with some identification of its vertices with $\mathcal{F}$. Take some large enough ball $B \subset \mathbb{R}^{d}$, containing all the sets of $\mathcal{F}$ in its interior. The configuration space that we study is $\Delta_{\Delta}^{r} \times B$, denote its elements by $\left(\alpha_{1}, \alpha_{2}, \ldots, \alpha_{r}, p\right)$. The points $\alpha_{i}$ in the simplex $\Delta$ will be considered as functions $\alpha_{i}: \mathcal{F} \rightarrow \mathbb{R}^{+}$with unit sum.

Denote for brevity $\mathbb{R}^{d}=V$. Now let us map our configuration space to $V^{r}$ by the following rule. Let $\pi_{K}(p)$ be the orthogonal projection of $p$ to $K \in \mathcal{F}$. Put

$$
f\left(\alpha_{1}, \alpha_{2}, \ldots, \alpha_{r}, p\right)=\bigoplus_{i=1}^{r} \sum_{K \in \mathcal{F}} \alpha_{i}(K)\left(\pi_{K}(p)-p\right),
$$

This map is evidently continuous and $G$-equivariant, if we identify $V^{r}$ with $V[G]$ ( $V$-valued functions on $G$ with $G$-action by right multiplication by $g^{-1}$ ).

Denote the zero set of $f$ by $Z$. Similar to [11, the map $f$ can be considered as a section of $G$-equivariant vector bundle, its Euler class being

$$
e(f)=w^{d} \times u \in H_{G}^{r d}\left(\Delta_{\Delta}^{r} \times B, \Delta_{\Delta}^{r} \times \partial B\right),
$$


where $w$ is the image of the $e(I[G]), u$ is the generator of $H^{d}(B, \partial B)$. By Lemmas 1 and 3, $w^{d} \neq 0 \in H_{G}^{d(r-1)}\left(\Delta_{\Delta}^{r}\right)$, and $e(f) \neq 0$.

Similar to the proof of Lemma 3 in [11], we conclude that the natural map $A_{G}^{l} \rightarrow H_{G}^{l}(Z)$ is injective in dimensions $l \leq n-r-(r-1) d=n-1-(r-1)(d+1)$. Let us sketch the proof of this claim. Suppose that some $\xi \in A_{G}^{l}$ maps to zero in $H_{G}^{l}(Z)$ by the natural map $\pi_{Z}^{*}: A_{G}^{*} \rightarrow H_{G}^{*}(Z)$, then by the properties of the cohomology multiplication

$$
\xi w^{d} \times u=0 \in H_{G}^{r d+l}\left(\Delta_{\Delta}^{r} \times B, \Delta_{\Delta}^{r} \times \partial B\right),
$$

which contradicts with Lemma 3 ,

It follows from the cohomology lower bound on the genus that $\mathrm{g}_{\text {free }}(Z) \geq n-(r-1)(d+$ $1) \geq c+1$. Now we are going to use this fact and show that the point $p$ is not contained in any $K \in \mathcal{F}$ with $\alpha_{i}(K)>0$.

We can find small enough $\varepsilon>0$ so that the family of $\varepsilon$-neighborhoods $\mathcal{F}(\varepsilon)=\{K(\varepsilon)\}_{K \in \mathcal{F}}$ has covering multiplicity at most $c$. Now consider the following open subsets of $Z$ : for any $K \in \mathcal{F}$ denote

$$
U_{K}=\left\{\left(\alpha_{1}, \alpha_{2}, \ldots, \alpha_{r}, p\right) \in Z: \exists i \in[r] \text { such that } \alpha_{i}(K)>0 \text { and } p \in K(\varepsilon)\right\} .
$$

Note that for any $\left(\alpha_{1}, \alpha_{2}, \ldots, \alpha_{r}, p\right) \in U_{K}$ there is only one $i \in[r]$ such that $\alpha_{i}(K)>0$, since we consider the deleted product $\Delta_{\Delta}^{r}$. Hence the set $U_{K}$ is partitioned into connected components, that are permuted by $G$ freely, i.e. it is inessential. The family $\left\{U_{K}\right\}$ covers $Z$ with multiplicity at most $c$. If it does cover $Z$, than $g_{\text {free }}(Z) \leq c$, that was shown above to be false.

Therefore, there exists a combination $\left(\alpha_{1}, \alpha_{2}, \ldots, \alpha_{r}, p\right)$ with the following property: if $\alpha_{i}(K)>0$, then $p \notin K(\varepsilon)$. Put

$$
\mathcal{F}_{i}=\left\{K \in \mathcal{F}: \alpha_{i}(K)>0\right\},
$$

the families $\mathcal{F}_{i}$ are disjoint. For any $i \in[r]$ the point $p$ is in the convex hull of the points $X_{i}=\left\{\pi_{K}(p)\right\}_{K \in \mathcal{F}_{i}}$, reducing the family $\mathcal{F}_{i}$ if needed, we may assume that $p$ is in the relative interior of $X_{i}$. It is clear, that for any ray $\rho$ starting at $p$, some of the angles $\angle\left(\rho, \pi_{K}(p)-p\right)\left(K \in \mathcal{F}_{i}\right)$ is at least $90^{\circ}$, and $\rho$ cannot intersect the corresponding set $K$.

\section{Proof of Corollary 2}

If $r$ is a prime power, then the statement follows from Theorem 1, Otherwise choose a positive integer $k$ so that $R=k(r-1)+1$ is prime, such $k$ exists by the Dirichlet theorem on arithmetic progressions. Now consider the family $\mathcal{G}$ of $k n$ sets, that is obtained from $\mathcal{F}$ by taking each member of $\mathcal{F}$ exactly $k$ times. Any point in $\mathbb{R}^{d}$ belongs to at most $k c$ sets of $\mathcal{G}$. The inequality

$$
k n \geq(d+1)(R-1)+k c+1=k(d+1)(r-1)+k c+1
$$

holds since $k n \geq k(d+1)(r-1)+k c+k$. Hence there exists a point $p \in \mathbb{R}^{d}$ such that any ray $\rho$ starting at $p$ does not intersect at least $R$ members of $\mathcal{G}$, In this case it is clear that $\rho$ does not intersect at least $r$ members of $\mathcal{F}$. 


\section{Proof of Theorem 3}

The proof mainly follows the proof of Theorem 1, though some changes are required. Denote again

$$
f\left(\alpha_{1}, \alpha_{2}, \ldots, \alpha_{r}, p\right)=\bigoplus_{i=1}^{r} \sum_{K \in \mathcal{F}} \alpha_{i}(K)\left(\pi_{K}(p)-p\right),
$$

to use the above reasonings, the map $f$ should not have zeros on $\Delta_{\Delta}^{r} \times \partial B$ for large enough ball $B$. But in the case of hyperplanes this is not true. We need the following lemma from [1].

Lemma 5. Suppose $\mathcal{F}=\left\{h_{1}, \ldots, h_{n}\right\}$ is a set of hyperplanes in $\mathbb{R}^{d}$, consider the orthogonal projections $\pi_{1}, \ldots, \pi_{n}$ onto the respective hyperplanes. Then there exists a convex body $P$, such that

$$
\forall i=1, \ldots, n, \pi_{i}(P) \subseteq P .
$$

Take the convex body $P$ from Lemma 5. Denote the zero set of $f$ on $\Delta_{\Delta}^{r} \times P$ by $Z$, this set still can have nonempty intersection with $\Delta_{\Delta}^{r} \times \partial P$.

Suppose that $P$ contains the origin, and approximate the map $f$ on $P$ by

$$
f_{\varepsilon}\left(\alpha_{1}, \alpha_{2}, \ldots, \alpha_{r}, p\right)=\bigoplus_{i=1}^{r} \sum_{K \in \mathcal{F}} \alpha_{i}(K)\left((1-\varepsilon) \pi_{K}(p)-p\right),
$$

denote its zero set by $Z_{\varepsilon}$. It is clear that

$$
Z_{\varepsilon} \cap \Delta_{\Delta}^{r} \times \partial P=\emptyset
$$

and, similar to the proof of Theorem 1, $\mathrm{g}_{\text {free }}\left(Z_{\varepsilon}\right) \geq c+1$.

Suppose that $\mathrm{g}_{\text {free }}(Z) \leq c$, then its open cover by $c$ inessential sets should be an open cover for $Z_{\varepsilon}$, for small enough $\varepsilon$. Hence, $\mathrm{g}_{\text {free }}\left(Z_{\varepsilon}\right) \leq c$, that is not true. Therefore, $\mathrm{g}_{\text {free }}(Z) \geq$ $c+1$, and the end of the reasoning is the same as in the proof of Theorem 1.

\section{REFERENCES}

[1] R. Aharoni, P. Duchet, B. Wajnryb. Successive projections on hyperplanes. // J. Math. Anal. Appl., 103, 1984, 134-138.

[2] I. Bárány, S.B. Shlosman, S. Szücz. On a topological generalization of a theorem of Tverberg. // J. London Math. Soc., II. Ser., 23, 1981, 158-164.

[3] T. Bartsch. Topological methods for variational problems with symmetries. Berlin-Heidelberg: Springer-Verlag, 1993.

[4] J. Eckhoff. Helly, Radon, and Carathéodory type theorems. // Handbook of Convex Geometry, ed. by P.M. Gruber and J.M. Willis, North-Holland, Amsterdam, 1993, 389-448.

[5] R. Fulek, A.F. Holmsen, J. Pach. Intersecting convex sets by rays. // Discrete and Computational Geometry, 42(3), 2009, 343-358.

[6] B. Grünbaum. Partitions of mass-distributions and of convex bodies by hyperplanes. // Pacific J. Math., 10, 1960, 1257-1261.

[7] A. Hatcher. Algebraic Topology. Cambridge University Press, 2002.

[8] E. Helly. Über Mengen konvexer Körper mit gemeinschaftlichen Punkten. // Jber Deutsch. Math. Verein., 32, 1923, 175-176.

[9] Wu Yi Hsiang. Cohomology theory of topological transformation groups. Springer Verlag, 1975. 
[10] R.N. Karasev. Dual theorems on central points and their generalizations. // Sbornik: Mathematics, 199(10), 2008, 1459-1479.

[11] R.N. Karasev. Analogues of the central point theorem for families with $d$-intersection property in $\mathbb{R}^{d}$. // arXiv:0906.2262v1, 2009.

[12] R.N. Karasev. Theorems of Borsuk-Ulam type for flats and common transversals (In Russian). // Sbornik: Mathematics, 200(10), 2009, 39-58; translated in arXiv:0905.2747v1.

[13] M.A. Krasnosel'skii. On the estimation of the number of critical points of functionals (In Russian). // Uspehi Mat. Nauk, 7(2), 1952, 157-164.

[14] G. Luke, A.S. Mishchenko. Vector bundles and their applications. Springer Verlag, 1998.

[15] J. Matoušek. Using the Borsuk-Ulam theorem. Berlin-Heidelberg, Springer Verlag, 2003.

[16] J. McCleary. A user's guide to spectral sequences. Cambridge University Press, 2001.

[17] J. Milnor, J. Stasheff. Characteristic classes. Princeton University Press, 1974.

[18] B.H. Neumann. On an invariant of plane regions and mass distributions. // J. London Math. Soc., 20, 1945, 226-237.

[19] R. Rado. A theorem on general measure. // J. London Math. Soc., 21, 1946, 291-300.

[20] P.J. Rousseeuw, M. Hubert. Depth in an arrangement of hyperplanes. // Discrete and Computational Geometry, 22, 1999, 167-176.

[21] A.S. Schwartz. Some estimates of the genus of a topological space in the sense of Krasnosel'skii. (In Russian) // Uspehi Mat. Nauk, 12:4(76), 1957, 209-214.

[22] A.S. Schwartz. The genus of a fibre space. // Trudy Moskov. Mat. Obsc., 11, 1962, 99-126; translation in Amer. Math. Soc. Trans., 55, 1966, 49-140.

[23] H. Tverberg. A generalization of Radon's theorem. // J. London Math. Soc., 41, 1966, $123-128$.

[24] A.Yu. Volovikov. On a topological generalization of the Tverberg theorem. // Mathematical Notes, $59(3), 1996,324-326$.

[25] A.Yu. Volovikov. On the Cohen-Lusk theorem (In Russian). // Fundamental and Applied Mathematics, 13(8), 2007, 61-67.

E-mail address: r_n_karasev@mail.ru

Roman Karasev, Dept. of Mathematics, Moscow Institute of Physics and Technology, Institutskiy PeR. 9, Dolgoprudny, Russia 141700 